\title{
DESCRIPTION AND INTERPRETATION OF SYSTEMATIC DEVIATIONS FROM EPITAXIAL LAWS OF OVERGROWTH*
}

\author{
H. Berger, P. Möck and B. Rosner \\ Humboldt-Universität zu Berlin, Institut für Kristallographie und Materialforschung \\ Invalidenstrasse 110, Unter den Linden 6, 10099 Berlin, Germany
}

(Received February 10, 1993; revised version May 5, 1993)

The effect of epitaxial misorientation on substrates liaving vicinal surfaces is studied using two simple geometrical models. The misorientations in two epitaxial systems, single layers and superlattices, can be partially explained by means of these models. Furthermore, the fundamentals of a new description tool for orientation relationships of epitaxial systems are outlined briefly, and two examples of application are given.

PACS numbers: 68.55.-a, 68.65.+g

\section{Introduction}

From the geometrical point of view epitaxial systems are commonly characterized by simple epitaxial laws $\left((h k l)\right.$ of deposit parallel to $\left(h^{\prime} k^{\prime} l^{\prime}\right)$ of substrate; $[u v w]$ of deposit parallel to $\left[u^{\prime} v^{\prime} w^{\prime}\right]$ of substrate). llowever, there is a lot of experimental evidence (see, e.g., [1-14]) that the epitaxial laws are an oversimplification not suitable for the detailed description of structural relations between substrate and deposit and, therefore, of their correlation with the growth process and physical properties.

A frequent origin for deviations from simple overgrowth relations is the cut-off of the substrate surface off high-symmetric net planes (vicinal surfaces), which leads to additional tilt of the deposit. A simple geometrical model has been suggested by Nagai [1] and by Pond et al. [2] in order to understand this tilt effect. Reviews concerning different reasons of epitaxial misorientation have been published recently by Aindow and Pond [3] and by Möck [15].

The aim of this paper is a discussion and a comparison of epitaxial misorientation on miscut substrates, which partially have been published earlier [13, 14],

* This paper is dedicated to Professor Dr. Julian Aulcytncr on the occasion of his 70th birthday. 
calculated with the help of simple geometrical models. Single layers and superlattices are considered.

Furthermore, a recently developed description tool for orientation relationships of epitaxial systems using transformation matrices is described briefly. Results of the application of this description tool will be also given.

\section{Overgrowth models}

Two simple models of epitaxial overgrowth on vicinal substrate surfaces, which may be regarded as geometrical limiting cases, are shown in Figs. 1 and 2. Model I discussed firstly by Nagai [1] is sketched in a simplified manner. The atomic configurations around the steps in the interface are accommodated and
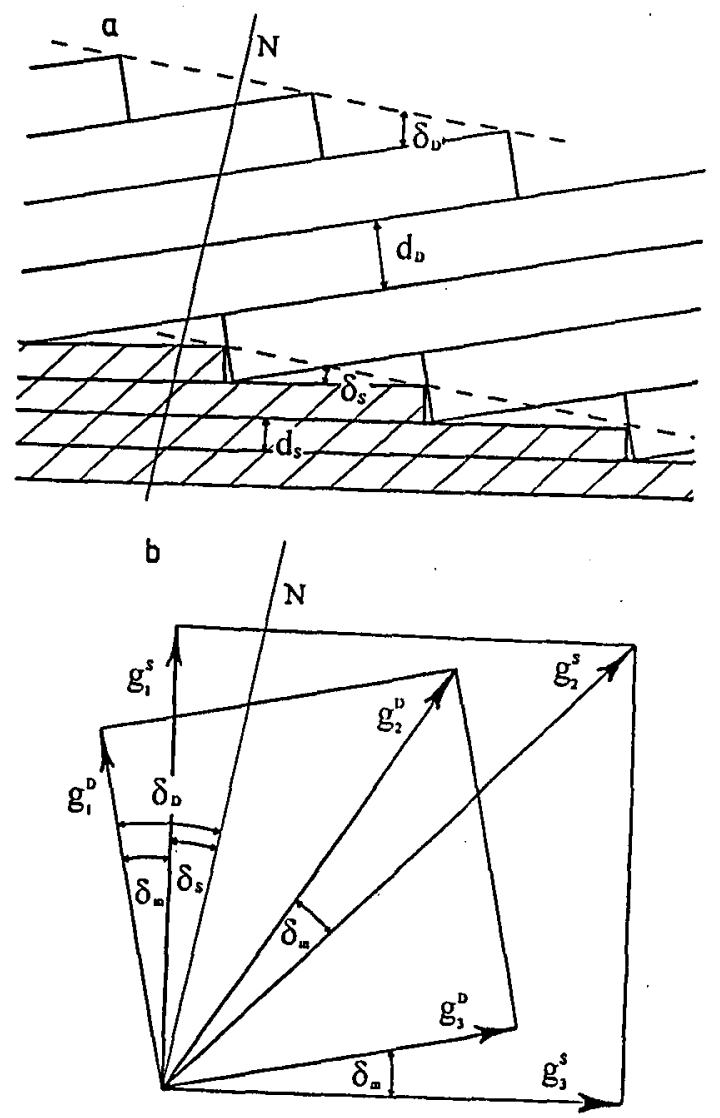

Fig. 1. Model I: misoriented deposit (D) on a miscut substrate (S). (a) Structural model. $d_{\mathrm{D}}, d_{\mathrm{S}}-$ monolayer thicknesses (net-plane distances); $\delta_{\mathrm{D}}, \delta_{\mathrm{S}}$ - tilt angles of the monolayers to the surface of the epitaxial sample. (b) Cubic unit cells (direct or reciprocal space) in the tilt plane. $g_{1,2,3}$ - (reciprocal) lattice vectors; $N$ - surface normal. 

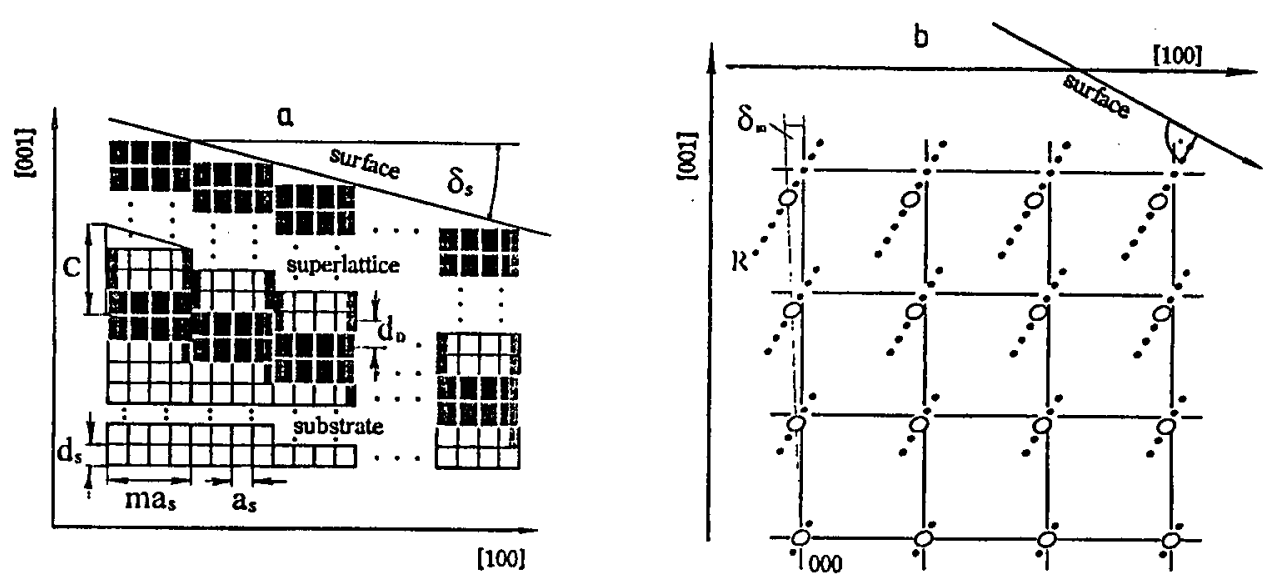

Fig. 2. Model II: parallel overgrowth of the deposit (superlattice) on a miscut substrate (cubic metrics; see Fig. 1 for symbols). (a) Structural model. Monoclinic supercell marked $\left(C, m a_{s}\right)$. (b) Reciprocal lattices of substrate and deposit in the tilt plane. o reciprocal lattice points corresponding to the main peak or the peak of the average lattice, respectively; - reciprocal lattice points corresponding to thickness oscillations or superlattice peaks, respectively.

can be regarded as partial dislocations (interfacial dislocations) occurring additionally to dislocations relieving lateral mismatch (misfit dislocations). The misfit perpendicular to the surface leads to an additional misorientation of the deposit. For substrate cut-ofls up to a few degrees there exists the simple relation

$$
\delta_{\mathrm{D}} / \delta_{\mathrm{S}}=d_{\mathrm{D}} / d_{\mathrm{S}},
$$

where $\delta_{\mathrm{D}}, \delta_{\mathrm{S}}$ are the tilt angles of deposit and substrate to the surface of the epitaxial sample, and $d_{\mathrm{D}}, d_{\mathrm{S}}$ are the corresponding monolayer thicknesses (net plane distances) - see Fig. 1a. The lattice of the deposit is rigidly rotated with respect to that of the substrate. For cubic metrics of both lattices and negligible distortions of deposit and substrate, the angle differences $\delta_{\mathrm{m}}=\delta_{\mathrm{D}}-\delta_{\mathrm{S}}$ (misorientation angles) are the same for any corresponding crystallographic directions of both lattices (Fig. 1b).

Model II implies parallel overgrowth of the deposit [14]. This model is less probable for thicker single layers and is discussed only in connection with the overgrowth of superlattices (Fig. 2a). In model II the nature of the atomic arrangement at the steps in every monolayer is not considered and therefore in this model, lateral misfit is not included. In order to understand the diffraction properties of such an equally spaced stair-like layer system, the analytical expression for the diffracted intensity according to the kinematic theory of diffraction can be used [14]. It yields that maximum intensity is obtained for rows $R$ in the reciprocal lattice running through the reciprocal lattice points of the substrate perpendicular to the surface (Fig. 2b). Some geometrical features of this result can be seen also from the relation between direct and reciprocal unit supercells, presented in 
Fig. 2a. For a single layer the thickness oscillations occur along the row $R$, where the position of the point corresponding to the peak intensity is determined by the monolayer distance $d_{\mathrm{D}}$. It becomes evident that model II leads to (at most) monoclinic metrics of the layer. It should be noted that within the validity of the model the same expression (1) holds for the angle $\delta_{\mathrm{m}}$ which is measured as the misorientation between the substrate and the average lattice of the deposit using nearly symmetric reflections. The reciprocal lattice points corresponding to the superlattice peaks lie on the row $R$, and the above considerations are valid for any of these reciprocal lattice vectors including that of the average lattice.

The reciprocal lattice relations characteristic of model II remain valid also for inclined monolayers of the deposit as far as one supercell edge is perpendicular to the substrate lattice planes which are nearly parallel to the surface. This is not very probable, but could be fulfilled statistically.

\section{Exact description of the orientation relationships}

For an exact description of the orientation relationships of epitaxial samples, transformation matrices can be used [13]. These matrices transform the (average) lattice of the substrate (coordinate system $A$ ) into the (average) lattice of the deposit (coordinate system $B$ ) and vice versa. Using the notation of Bowles and Mackenzie (introduced and extensively described in Ref. [16] and also used in many textbooks, see, e.g., [17]) the transformation matrix

$$
(B N A)=\left(\begin{array}{lll}
N_{11} \pm \Delta N_{11} & N_{12} \pm \Delta N_{12} & N_{13} \pm \Delta N_{13} \\
N_{21} \pm \Delta N_{21} & N_{22} \pm \Delta N_{22} & N_{23} \pm \Delta N_{23} \\
N_{31} \pm \Delta N_{31} & N_{32} \pm \Delta N_{32} & N_{33} \pm \Delta N_{33}
\end{array}\right)
$$

transforms the direct lattice of the substrate into the direct lattice of the deposit. The components $\Delta N_{i j}$ are the error limits, which depend on the precision and accuracy of the experimental methods.

The transformation matrices characterize entirely the epitaxial system from the lattice geometrical point of view. For a survey of the different effects of the lattice transformation, these matrices can be divided into several factors using the experimental results and the rotation matrix of the ideal orientation relationship. For example, these factors are:

- the scalar of the volume change [e.g. $|(B N A)|]$,

- the deformation tensor of the deposit [e.g. $(B D B)]$,

- the rotation matrix of epitaxial misorientation [e.g. $(B A B)$ ],

- the matrix of the ideal orientation relationship [e.g. $(B I A)]$, and

- the deformation tensor of the substrate [e.g. $(A C A)]$.

For the transformation from the direct lattice of the substrate into the direct lattice of the deposit one obtains

$$
(B N A)=\sqrt[3]{|(B N A)|}(B D B)(B A B)(B I A)(A C A) .
$$

From the rotation matrix of epitaxial misorientation, the angle of misorientation

$$
\delta_{\mathrm{m}}=\arccos \left[\frac{1}{2}\left(\sum A_{i i}-1\right)\right]
$$


and the axis of misorientation

$$
r_{i}=\frac{A_{j k}-A_{k j}}{2 \sin \delta_{\mathrm{m}}}, \quad i, j, k \text { cyclic, }
$$

can be derived.

The rotation matrix of the ideal orientation relationship contains the same information as the law of overgrowth.

The transformation matrices can be further used to calculate the admissible line defects at the interface using Pond's theory [18], to derive the symmetry group of the deformed deposit, and to deduce information concerning lattice geometry.

A detailed explanation of this description tool is given by Möck [15].

\section{Discussion of some examples}

The angles $\delta_{\mathrm{D}}$ as a function of the vicinal angles $\delta_{\mathrm{S}}$ on CdTe (111) deposited on miscut (001) GaAs, which were measured by X-ray difrractometry on selected samples, are shown in Fig. 3. The constant ratio $\delta_{\mathrm{D}} / \delta_{\mathrm{S}}=1.32$ obeys relation (1).

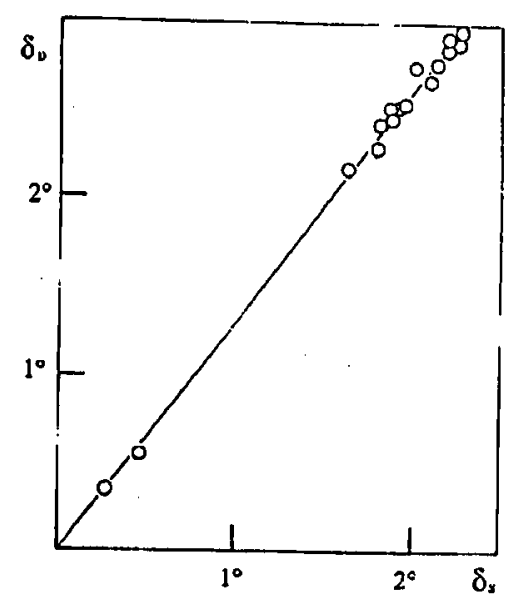

Fig. 3. Measured tilt angles $\delta_{D}$ of the deposit vs. vicinal angles $\delta_{S}$ of the substrate. CdTe(111)/GaAs (001); full line $-\delta_{\mathrm{D}}=1.32 \delta_{\mathrm{S}}$.

Thus the rigid model I should be valid as supposed in principle also by some other authors for this $[11,12]$ and other material systems [1-10]. On the other hand, for a number of specimens there resulted other, smaller as well as greater values of $\delta_{\mathrm{D}} / \delta_{\mathrm{S}}$.

Apparently, the overgrowth according to model I corresponds to a state which is dominated only by geometrical conditions. Further parameters, connected with the growth process, may lead to deviations from these conditions and, therefore, influence the actual overgrowth relationships.

For two epitaxial specimens of CdTe on GaAs (substrate miscut $2^{\circ}$ around [110]), one in agreement, one in disagreement with model I, the transformation 
matrices determined by X-ray goniometry [13] are given below. The transformation matrix of the sample 1 ,

$$
(B N A)_{1}=\left(\begin{array}{rrr}
-0.1806 \pm 0.002 & 0.6915 \pm 0.004 & -0.4997 \pm 0.003 \\
0.6916 \pm 0.004 & -0.1807 \pm 0.002 & -0.4998 \pm 0.003 \\
-0.4995 \pm 0.003 & -0.4997 \pm 0.003 & -0.5109 \pm 0.004
\end{array}\right)
$$

is significantly different from that of sample 2 ,

$$
(B N A)_{2}=\left(\begin{array}{rrr}
-0.1756 \pm 0.002 & 0.6964 \pm 0.003 & -0.4997 \pm 0.003 \\
0.6968 \pm 0.003 & -0.1757 \pm 0.002 & -0.4942 \pm 0.003 \\
-0.4945 \pm 0.003 & -0.4948 \pm 0.003 & -0.5209 \pm 0.003
\end{array}\right) .
$$

The decomposition of the transformation matrices leads to the conclusion that the epitaxial samples differ mostly in the rotation matrices of epitaxial misorientation. The rotation parameters were derived from these matrices and are given in Table. Within the range of the error limits, the samples differ only in the angles of epitaxial misorientation. Following Pond's theory of line defects in interfacial

TABLE

\begin{tabular}{|c|c|c|}
\hline $\begin{array}{c}\text { Sample } \\
\text { No. }\end{array}$ & $\begin{array}{c}\text { Angle of epitaxial } \\
\text { misorientation } \\
\delta_{\mathrm{m}}\left[^{0}\right]\end{array}$ & $\begin{array}{c}\text { Axis of epitaxial } \\
\text { misorientation } \\
{\left[ \pm 0.05^{\circ}\right]}\end{array}$ \\
\hline 1 & $0.60 \pm 0.04$ & {$\left[\begin{array}{lll}71 & 70 & 0\end{array}\right]$} \\
\hline 2 & $1.41 \pm 0.04$ & {$\left[\begin{array}{lll}\overline{72} & 70 & 0\end{array}\right]$} \\
\hline
\end{tabular}

Rotation parameters of epitaxial misorientation [13].

dislocations exist, as discussed elsewhere [13]. On the other hand, according to this theory, one can expect that in sample 2 in addition to the common interfacial and misfit dislocations other crystal defects are present.

The calculation of the symmetry group of the deformed deposits gives the point symmetry groups $m$ or 1 corresponding to monoclinic or triclinic symmetry.

An example of epitaxial overgrowth which corresponds to model II has been found at a superlattice of $\left(\mathrm{In}_{\mathrm{n}}, \mathrm{Ga}\right) \mathrm{As} / \mathrm{GaAs}$ on $\mathrm{Ga \Lambda s}$ deposited on miscut (001) $\mathrm{GaAs}$ [14]. In X-ray rocking curves, substrate peak and superlattice peaks near the substrate peak do not change the position on varying the azimuth of incidence (Fig. 4). This means that the reciprocal lattice row of the superlattice runs through the reciprocal lattice point of the substrate as suggested by model II. The angle distances of the superlattice peaks are changed corresponding to the predictions of the model.

The diffraction patterns of an analogous superlattice specimen, which cannot be interpreted by means of models I and II is shown in Fig. 5. From the distance of the substrate and the average-lattice peaks it follows that there exists an epitaxial misorientation much greater than that predicted by the geometrical models $\left(d_{\mathrm{D}} / d_{\mathrm{S}}=1.01 ; \delta_{\mathrm{D}} / \delta_{\mathrm{S}} \approx 1.24\right)$. Therefore, in this case the substrate miscut cannot be the immediate reason of the deposit misorientation. 


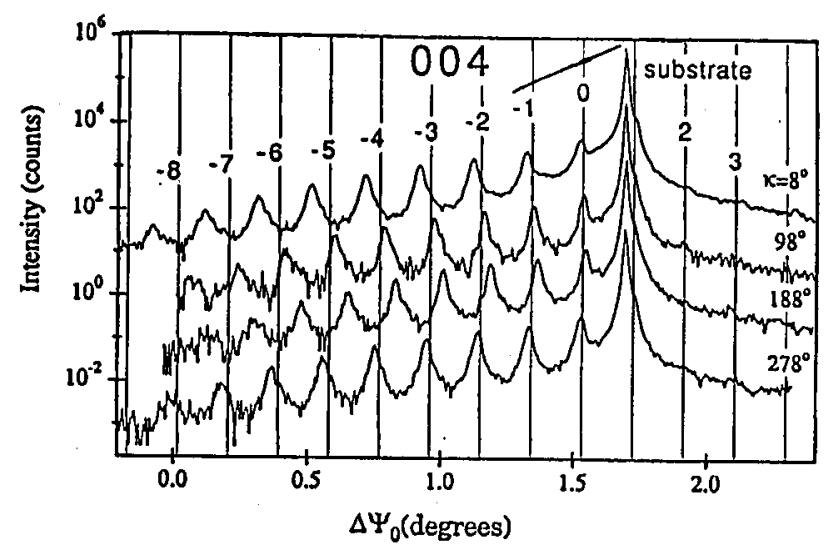

Fig. 4. X-ray rocking curves of an ( $\left.\mathrm{I}_{1}, \mathrm{Ga}\right) \mathrm{As} / \mathrm{GaAs}(001)$ superlattice on $\mathrm{GaAs}(001)$ [14]. Reflection group 004 at four azimuths of incidence, $\kappa$ (diflerence $90^{\circ}$ ). Superlattice period $27.5 \mathrm{~nm}, 10$ periods, vicinal angle $2.6^{\circ} ; \mathrm{Cu} K_{\alpha_{1}} ; \Delta \Psi_{0}-$ angle of incidence (normalized for equal substrate peak position).

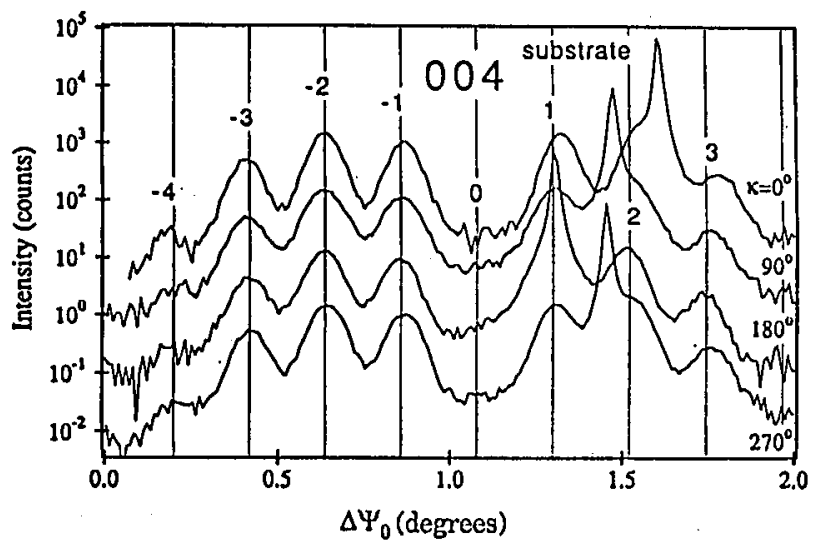

Fig. 5. X-ray rocking curves of an (In, Ga)As/GaAs (001) superlattice on GaAs (001) [14]. Reflection group 004 at four azimutls of incidence, $\kappa$ (difference $90^{\circ}$ ). Superlatlice period $23.7 \mathrm{~nm}, 30$ periods, vicinal angle $0.6^{\circ} ; \mathrm{Cu} K_{a_{1}} ; \Delta \Psi_{0}-$ angle of incidence (normalized for equal superlattice peak positions near the substrate peak).

\section{Conclusions}

The experimental examples selected from two different single and one multilayer epitaxial systems have shown that in certain cases simple geometrical models can be useful to interpret the effect of epitaxial misorientation. Ilowever, in both systems examples exist which cannot be interpreted on the basis of the considered models. Obviously, the whole phenomenon of epitaxial misorientation is a conse- 
quence of the accommodation of the deposit latlice to that of the substrate. This is a complex process which includes elastical distortion of the deposit lattice, vicinal step-related interfacial dislocations and further crystal defects. The question, under which conditions the simple geometrical models are valid, demands more systematic studies in dependence on geometrical and growth parameters. Characterization of epitaxial systems by means of transformation matrices can be a helpful tool for such investigations.

\section{References}

[1] H. Nagai, J. Appl. Phys. 45, 3789 (1974).

[2] R.C. Pond, M. Aindow, C. Dineen, T. Petcrs, Inst. Phys. Conf. Ser. 87, 181 (1987).

[3] M. Aindow, R.C. Pond, Philos. Mag. A 63, 667 (1991).

[4] W.J. Bartels, W. Nijman, J. Cryst. Growth 44, 518 (1978).

[5] A. Olki, N. Slibata, S. Zcmbutsu, J. Appl. Phys. 64, 694 (1988).

[6] J. Kleiman, R.M. Park, II.A. Mar, J. Appl. Phys. 64, 1201 (1988).

[7] P. Auvrey, M. Baudet, A. Regreny, J. Cryst. Growth 95, 288 (1989).

[8] P. Maigne, A.P. Roth, J. Cryst. Crowll 118, 117 (1992).

[9] P. Maigne, A.P. Roth, Semicond. Sci. Technol. 7, 1 (1992).

[10] A. Lieberich, J. Levkoff, J. Cryst. Growth 100, 330 (1990).

[11] S. Tatarenko, J. Cibert, Y. Gobil, G. Fcuillet, E. Ligeon, L.S. Dang, K. Saminadayar, J. Cryst. Growth 101, 126 (1990).

[12] E. Ligeon, C. Chami, R. Daniclou, G. Fcuillct, J. Fontenille, K. Saminadayar, A. Ponchet, J. Cibert, Y. Gobil, S. Tatarenko, J. Appl. Phys. 67, 2428 (1990).

[13] P. Möck, J. Mater. Sci. Eng. B 16, 165 (1993).

[14] B. Rosner, H. Berger, J. Appl. Crystallogr., 1993, in press.

[15] P. Möck, J. Cryst. Growth 128, 122 (1993).

[16] J.M. Bowles, J.K. Mackenzie, Acla Mctall. 2, 129 (1951).

[17] J.W. Christian, The Theory of Transformation in Melals and Alloys, Pergamon Press, Oxford 1965.

[18] R.C. Pond, in: Dislocations in Solids, Vol 8, Ed. F.R.N. Nabarro, Elsevier, Amsterdam 1989, p. 1. 